\title{
Effects of Ferric Citrate Hydrate on Calciprotein Particle Levels and Aortic Arch Calcification Progression in Patients on Maintenance Hemodialysis: A Comparison with Calcium Carbonate
}

\author{
Kenichi Akiyama',2, Makoto Kuro-0², Yutaka Miura², Hiroshi Kawaguchi ${ }^{3}$, Kosaku Nitta1 \\ ${ }^{1}$ Department of Nephrology, Tokyo Women's Medical University, Tokyo, Japan \\ ${ }^{2}$ Division of Anti-Aging Medicine, Center for Molecular Medicine, Jichi Medical University, Tochigi, Japan \\ ${ }^{3}$ Department of Nephrology, Jyoban Hospital, Fukushima, Japan \\ Email:knitta@twmu.ac.jp
}

How to cite this paper: Akiyama, K., Kuro-O, M., Miura, Y., Kawaguchi, H. and Nitta, K. (2020) Effects of Ferric Citrate Hydrate on Calciprotein Particle Levels and Aortic Arch Calcification Progression in Patients on Maintenance Hemodialysis: A Comparison with Calcium Carbonate. International Journal of Clinical Medicine, 11, $1-14$.

https://doi.org/10.4236/ijcm.2020.111001

Received: November 17, 2018

Accepted: December 30, 2019

Published: January 2, 2020

Copyright $\odot 2020$ by author(s) and Scientific Research Publishing Inc. This work is licensed under the Creative Commons Attribution International License (CC BY 4.0).

http://creativecommons.org/licenses/by/4.0/ (c) (i) Open Access

\begin{abstract}
Background: Arterial calcification is a predictive marker in patient on hemodialysis (HD), but the relationship between arterial calcification and calciprotein particles (CPPs) is unclear. Methods: We examined the effects of ferric citrate hydrate (JTT-751) on CPP level and evaluated changes in aortic arch calcification (AoAC) grade in patients on maintenance HD (MHD). In total, 70 MHD patients were enrolled in the study and followed for 24 months. We measured serum CPP levels and fibroblast growth factor 23 (FGF 23) among propensity score-matched MHD patients. One group ( $\mathrm{n}=$ 35) was treated with $\mathrm{CaCO}_{3}$ and the other $(\mathrm{n}=35)$ was treated with ferric citrate hydrate (JTT-751). AoAC was assessed on chest-X rays. Eligible patients continued the same treatment. Results: All 70 patients completed the study. Serum CPP levels reduced in the JTT-751 group, but were not significantly different in the $\mathrm{CaCO}_{3}$ group. Among patients whose baseline AoAC score (AoACS) was $\leq 4$ (median), median AoACS increased from $0(0-3)$ to 3 (2 4) $(\mathrm{p}<0.05)$ and $\triangle \%$ AoACS was $2(0 \%-4 \%)$ in the JTT-751 group. In the $\mathrm{CaCO}_{3}$ group, median AoACS increased from $2(0-2)$ to $3(0-4)(\mathrm{p}<0.05)$ and $\Delta \%$ AoACS was $1(0 \%-3 \%)$. Conclusion: These results indicate that the administration of JTT-751 decreased serum CPP levels but did not inhibit AoAC progression in patients on MHD.
\end{abstract}

\section{Keywords}

Hemodialysis, Ferric Citrate Hydrate, Calciprotein Particles, Vascular 


\section{Introduction}

High serum phosphate $(\mathrm{P})$ level is a frequent manifestation in advanced chronic kidney disease $(\mathrm{CKD})$ and an important factor for CKD-mineral bone disorder (CKD-MBD) [1]. Taniguchi et al. showed an increased mortality risk linked to hyperphosphatemia among patients on hemodialysis (HD) [2]. The use of $\mathrm{P}$ binders was found to be associated with decreased mortality when contrasted with a no-treatment group [3]. However, $\mathrm{P}$ binders place a considerable burden on patients due to the high number of pills, which leads to low adherence reduced health-related quality of life [4]. Non-calcium (Ca)-based P binders have been recommended to facilitate better prognosis of patients on HD by reducing vascular calcification grade [5].

Calciprotein particles (CPPs) are a complex of $\mathrm{Ca}, \mathrm{P}$, and fetuin- $\mathrm{A}$ and have the vital physiological function of transporting hydroxyapatite into bone [6]. With excessive $\mathrm{P}$ and $\mathrm{Ca}, \mathrm{CPP}$ levels in blood are elevated, and this is contributory to arteriosclerosis [7] and inflammation [8] in patients with CKD. CPP levels correlate significantly with serum levels of $P$ and fibroblast growth factor 23 (FGF23), but not with Ca levels, and therefore can increase in CKD patients with hyperphosphatemia or hypocalcemia [9].

A newly developed Ca-free P binder, ferric citrate hydrate (JTT-751), is currently undergoing development as a therapy for hyperphosphatemia in CKD patients. While ferric citrate is a versatile agent with various uses such as a food additive and has been reported to reduce serum P levels [10], JTT-751 is a novel bioactive pharmaceutical agent with a greater surface area than conventional ferric citrate, which confers it with a more favorable dissolution rate. These features may factor in the strong ability of JTT-751 to bind P in the gastrointestinal tract. Indeed, JTT-751 has shown an excellent serum P-reducing effect without serious side effects at relatively low doses in HD patients [11] [12].

This study sought to compare the effects of JTT-751 with those of a Ca-based $P$ binder on aortic calcification (AoAC) and serum CPP levels in patients on maintenance HD (MHD).

\section{Methods}

\subsection{Study Subjects}

We calculated that the required number of subjects would be 70 for the two groups combined if the statistical power is $60 \%$, the one-sided level of significance was $5 \%$. A total of 70 eligible patients from the dialysis center of Jyoban Hospital were enrolled in this study in July 2015. Inclusion criteria were age $\geq 20$ years, a minimum of 3 months of stable condition, and at least 6 months on HD treatment. Exclusion criteria were severe gastrointestinal disease, hepatitis 
and/or liver cirrhosis, high risk of hemorrhage, hypocalcemia with an adjusted serum $\mathrm{Ca}$ of $<7.5 \mathrm{mg} / \mathrm{dL}$, severe cardiovascular dysfunction, poorly controlled diabetes mellitus (DM), meeting any of the contraindications for using JTT-751 or Ca-based $\mathrm{P}$ binders, and being considered unfit to participate by investigators. Also excluded were patients undergoing in-center nocturnal HD (extended-hours), those scheduled to undergo parathyroidectomy or live renal transplant within 6 months of enrollment, or others with an estimated life expectancy of $<3$ months.

All participants gave their written informed consent. The study protocol was approved by the Jyoban Hospital Institutional Ethical Committee (Approval no. 26-1) and was carried out in adherence to the tenets of the Declaration of Helsinki and the Committee on Human Research.

\subsection{Study Protocol}

All patients were randomized at a 1:1 ratio to either of two groups: the JTT-751 group (Riona $250 \mathrm{mg}$; Torii, Tokyo, Japan) or the calcium carbonate $\left(\mathrm{CaCO}_{3}\right)$ group (Caltan $500 \mathrm{mg}$; Merkhoei-Fuso, Tokyo, Japan). Each tablet was administered orally 3 times daily with meals for 24 months. At screening, patients were further grouped by age and sex with propensity score-matching. After screening, participants were not subjected to a washout period of withdrawal of all P binders. Due to the sample size and appearance and taste of the tablets, the participants, investigators, and attending physicians were not blinded to the study. However, outcome investigators were blinded to the assigned treatment and patient characteristics.

Following propensity score-matching, subjects were first given either JTT-751 or $\mathrm{CaCO}_{3}$ and the dose was titrated every 2 weeks for the first 6 weeks to allow for normalization of serum P levels $(4.5-5.5 \mathrm{mg} / \mathrm{dL}$ ) and correction of serum Ca levels $(8.5-10.5 \mathrm{mg} / \mathrm{dL})$. During the study period, dosage of the vitamin D analogue calcitriol was adjusted in line with usual practice for optimal management of patients with CKD-MBD. No subjects were administered aluminum-based $\mathrm{P}$ binders. We used the Japanese Society for Dialysis Therapy recommended target levels for serum biomarkers of CKD-MBD.

HD sessions were carried out using hollow-fiber dialyzers, such as cellulose triacetate and polysulfone, 3 times per week for $4 \mathrm{~h}$ daily). The set parameters were as follows: blood flow rate at $200 \mathrm{~mL} / \mathrm{min}$ and dialysate flow rate at 500 $\mathrm{mL} / \mathrm{min}$. Mean residual urine volume was $<100 \mathrm{~mL} /$ day as previously described [13]. Safety assessment was performed using laboratory assessment and documentation of adverse events.

\subsection{Assessment of AoAC}

For all participants, chest radiographs were reviewed by 2 radiologists independently. Radiographs were evaluated for any features indicative of AoAC based on a scale previously reported by Ogawa et al. [13]. The scale with 16 equal sec- 
tors was placed on the aortic arch on a chest X-ray film, sectors with calcification were identified and counted, and the number of sectors was divided by 16 . The resulting value was then expressed as a percentage, and this was considered the aortic arch calcification score (AoACS). This value was considered an index of the presence of AoAC. In a previous study, we confirmed that AoACS showed high correlation with AoAC volume as assessed using multi-slice computed tomography $(r=0.635, p<0.001)[13]$.

\subsection{Laboratory and Hemodynamic Assessments}

Blood sampling was done before the start of each dialysis session. The levels of serum albumin, $\mathrm{Ca}, \mathrm{P}$, total cholesterol, high-density lipoprotein (HDL) cholesterol, triglyceride, C-reactive protein (CRP) and hemoglobin were measured with standard laboratory tests. For the analysis, we used the mean of 3 measurements taken within 3 months before chest radiography. Serum levels of intact by electro chemiluminescence immunoassay. Adjusted serum Ca levels were calculated using the formula [Ca + (4-albumin) $]$. Serum CPP levels were measured using OsteoSence $750 \mathrm{EX}$ fluorescent imaging agent as previously described [9]. Sandwich enzyme-linked immunosorbent assay for human FGF23 (Kainos Laboratories Inc., Tokyo, Japan) was used to measure serum FGF23 level. The assay evaluates biologically active, full-length FGF23 using two FGF23 monoclonal antibodies as previously described [14]. Urea kinetics was evaluated by measuring the $\mathrm{Kt} / \mathrm{V}$, which is a blood-based dialysis parameter that reflects dialysis adequacy [15]. We also assessed the normalized protein catabolism rate (nPCR), which is an indirect index of protein intake and was calculated as previously described [16].

All participants underwent routine clinical examination to evaluate clinical status before the HD session. Measurements for systolic and diastolic blood pressure (BP) were obtained using a conventional mercury sphygmomanometer with the patient in the supine position after $10-15$ min of rest; mean values for 1 month were used for analysis. Elevated blood pressure was defined as (a) pre-dialysis systolic BP $>140 \mathrm{mmHg}$, (b) diastolic $\mathrm{BP}>90 \mathrm{mmHg}$, or (c) use of any antihypertensive therapy.

\subsection{Outcome Measures}

We carried out evaluations of safety and efficacy every 4 weeks. In this study, the primary outcome was changed in the extent of AoAC from baseline to 24 months. The secondary outcome was changed in serum CPP level.

\subsection{Statistical Analysis}

Continuous variables are shown as means \pm standard deviation (SD) and categorical variables are shown as percentage. For nonparametric testing, Spearman's correlation analysis was used to assess the relationship among bone-derived biomarkers, clinical and biochemical factors, and AoACS. For comparing the 
extent of AoAC severity, the chi-square test was used for categorical variables, the one-way analysis of variance was used for continuous variables with normal distribution, and the Kruskal-Wallis test was used for continuous variables with skewed distribution. We also compared the levels of bone-derived biomarkers between the 2 groups using a post-hoc Bonferroni-corrected Mann-Whitney U test. All analyses were performed using JMP for Windows version 11 (SAS Institute Inc., Cary, NC). Statistical significance was set at a $\mathrm{p}$-value $<0.05$.

\section{Results}

Table 1 shows the demographics of the study population at baseline. In total, 70 patients who met the inclusion criteria were enrolled and randomized into 2 groups:35 patients were allocated to receive $\mathrm{CaCO}_{3}$ and 35 were allocated to receive JTT-751. Participants were followed up and at 24 months all were still enrolled in the trial and thus were entered into the analysis. Serial AoACS assessment was possible in all 70 patients.

There were no significant differences for age, sex, dialysis vintage, $\mathrm{Kt} / \mathrm{V}$, diabetes, body mass index, nPCR, corrected Ca, P, iPTH, hemoglobin, ferritin, albumin, CRP, total cholesterol, HDL cholesterol, triglycerides, or use of antihypertensives, vitamin $\mathrm{D}$, cinacalcet, or statins. No patients had received coumarin anticoagulant therapy. However, the JTT-751 group had significantly higher serum CPP levels compared with the $\mathrm{CaCO}_{3}$ group $(\mathrm{p}=0.0019)$. Log serum FGF23 levels showed a positive correlation with $\log$ serum CPP levels $(r=0.609, p<$ 0.001) (Figure 1).

Table 2 shows a comparison of changes in biochemical markers in the 2 patient groups during the study period. Similar serum P and corrected Ca levels were observed in both groups throughout the duration of the study. No significant differences were observed at follow-up at 24 months. Serum iPTH levels tended to increase in both groups at 24 months, but there were no significant differences between the groups. The $\mathrm{CaCO}_{3}$ group showed decreased serum HDL cholesterol levels, whereas the JTT-751 group showed increased levels. Serum FGF23 levels increased in the $\mathrm{CaCO}_{3}$ group but did not change significantly in the JTT-751 group. Notably, serum CPP levels decreased in the JTT-751 group, with no significant differences in the $\mathrm{CaCO}_{3}$ group. Furthermore, no significant differences were noted in other biochemical markers between the 2 groups. There were no cases of bone fracture, hypercalcemia, or cardiovascular events during the study. None of the patients discontinued the study medication due to adverse effects. JTT-751 was administered at a starting dose of 750 $\mathrm{mg} /$ day and had been increased to $1930 \pm 510 \mathrm{mg} /$ day at the end of the study. $\mathrm{CaCO}_{3}$ was administered at a starting dose of $1500 \mathrm{mg} /$ day and had been increased to $1730 \pm 450 \mathrm{mg} /$ day at the end of the study. Prescribed doses of vitamin $\mathrm{D}$ and cinacalcet did not significantly differ between the 2 groups (data not shown). 
Table 1. Baseline characteristics of the study population.

\begin{tabular}{|c|c|c|c|c|}
\hline & Total $(\mathrm{n}=70)$ & $\mathrm{CaCO}_{3}(\mathrm{n}=35)$ & JTT-751 $(\mathrm{n}=35)$ & $\mathrm{P}$-value \\
\hline Age, years & $76(67-80)$ & $76(67-82)$ & $76(67-79)$ & 0.8415 \\
\hline Male, \% & $38(54.3)$ & $19(54.3)$ & $19(54.3)$ & 1.0000 \\
\hline $\begin{array}{l}\text { Dialysis vintage, } \\
\text { months }\end{array}$ & $32(13-65)$ & $36(16-72)$ & $30(11-60)$ & 0.1902 \\
\hline $\mathrm{Kt} / \mathrm{V}$ & $1.51(1.29-1.70)$ & $1.54(1.30-1.71)$ & $1.44(1.26-1.7)$ & 0.4042 \\
\hline Diabetes, n (\%) & $36(51.4)$ & $16(45.7)$ & $20(57.1)$ & 0.3388 \\
\hline $\mathrm{CPP}, \mathrm{pg} / \mathrm{ml}$ & $\begin{array}{c}102,041 \\
(54,856-201,152)\end{array}$ & $\begin{array}{c}60,330 \\
(41,022-120,055)\end{array}$ & $\begin{array}{c}155,857 \\
(78,082-290,852)\end{array}$ & 0.0019 \\
\hline FGF23, pg/ml & $1939(603-5962)$ & $1876(486-4423)$ & $2580(830-6736)$ & 0.1805 \\
\hline BMI & $21.1(18.0-23.8)$ & $21(18.0-23.8)$ & $21.1(17.7-24.5)$ & 0.9953 \\
\hline nPCR & $0.82(0.72-0.93)$ & $0.79(0.70-0.91)$ & $0.84(0.76-0.96)$ & 0.3539 \\
\hline $\mathrm{Ca}, \mathrm{mg} / \mathrm{dl}$ & $8.4(8.0-8.7)$ & $8.4(8.0-8.6)$ & $8.5(8.1-9.0)$ & 0.1428 \\
\hline $\mathrm{P}, \mathrm{mg} / \mathrm{dl}$ & $4.7(4.2-5.6)$ & $4.6(3.7-5.7)$ & $4.7(4.4-5.5)$ & 0.4072 \\
\hline $\mathrm{iPTH}, \mathrm{pg} / \mathrm{ml}$ & $128(58-215)$ & $141(76-226)$ & $112(67-213)$ & 0.2387 \\
\hline Hemoglobin, \% & $10.9(10.0-11.8)$ & $10.8(9.8-11.8)$ & $11.0(10.3-11.8)$ & 0.2901 \\
\hline Albumin, g/dl & $3.6(3.4-3.9)$ & $3.6(3.3-3.8)$ & $3.6(3.4-3.9)$ & 0.7142 \\
\hline CRP, mg/dl & $0.12(0.05-0.30)$ & $0.07(0.03-0.22)$ & $0.16(0.06-0.41)$ & 0.1012 \\
\hline $\mathrm{TC}, \mathrm{mg} / \mathrm{dl}$ & $160(138-182)$ & $151(130-184)$ & $168(143-181)$ & 0.2401 \\
\hline HDL-C, mg/dl & $46(36-55)$ & $47(39-59)$ & $44(36-53)$ & 0.4413 \\
\hline $\mathrm{TG}, \mathrm{mg} / \mathrm{dl}$ & $84(56-115)$ & $83(56-104)$ & $101(68-145)$ & 0.1267 \\
\hline $\begin{array}{c}\text { Anti-hypertensives, } \\
\text { n (\%) }\end{array}$ & $27(38.6)$ & $11(31.4)$ & $16(45.7)$ & 0.2195 \\
\hline Vitamin D, n (\%) & $42(60.0)$ & $22(62.9)$ & $20(57.1)$ & 0.6256 \\
\hline Cinacalcet, n (\%) & $3(4.3)$ & $1(2.9)$ & $2(5.7)$ & 0.5551 \\
\hline Statin, n (\%) & $9(12.9)$ & $6(17.1)$ & $3(8.6)$ & 0.2841 \\
\hline
\end{tabular}

Data are expressed as median (interquartile ranges) or numbers (\%). BMI, body mass index; Ca, corrected calcium; CPP, calciprotein particle; CRP, C-reactive protein; FGF23, fibroblast growth factor 23; HDL-C, high-density lipoprotein cholesterol; iPTH, intact parathyroid hormone; nPCR, normalized protein catabolic rate; $\mathrm{P}$, phosphorus; $\mathrm{TC}$, total cholesterol; TG, triglycerides.

Table 3 shows the changes in AoACS from baseline in all 70 patients during the study period. Median (interquartile range [IQR]) AoACS increased from 4 $(0$ - 7$)$ to $5(3-9)$ and median (IQR) change in AoACS ( $\triangle \%$ AoACS was 1 ( $0 \%$ $3 \%$ ) in the JTT-751 group. In the $\mathrm{CaCO}_{3}$ group, median (IQR) AoACS increased from $2(0-5)$ to $3(2-5)$ and $\Delta \%$ AoACS was $0(0 \%-3 \%)$. There were no significant differences between the 2 groups $(\mathrm{p}=0.412)$.

Results stratified by baseline AoACS are also shown in Table 3. Among patients with advanced AoAC at the start of the study (initial AoACS value 4), median (IQR) AoACS increased from 7 (6 - 10) to 8 (6 - 11) and $\triangle \%$ AoACS (IQR) was $1(0 \%-2 \%)$ in the JTT-751 group. In the $\mathrm{CaCO}_{3}$ group, median (IQR) 
Table 2. Changes in biochemical markers during the study period.

\begin{tabular}{|c|c|c|c|}
\hline \multicolumn{4}{|c|}{$\mathrm{CaCO}_{3}$} \\
\hline & Baseline & 24 months & $\mathrm{P}$-value \\
\hline $\mathrm{P}$ & $4.6(3.7-5.7)$ & $5.6(5.1-6.2)$ & 0.0822 \\
\hline $\mathrm{Ca}$ & $8.4(8.0-8.6)$ & $8.6(8.2-8.9)$ & 0.1374 \\
\hline iPTH & $141(76-226)$ & $155(95-217)$ & 0.7881 \\
\hline Ferritin & $98(42-169)$ & $141(37-226)$ & 0.0523 \\
\hline Hemoglobin & $10.8(9.8-11.8)$ & $10.7(10.2-11.5)$ & 0.9272 \\
\hline Albumin & $3.6(3.3-3.8)$ & $3.6(3.2-3.8)$ & 0.8224 \\
\hline CRP & $0.07(0.03-0.22)$ & $0.10(0.05-0.39)$ & 0.7178 \\
\hline $\mathrm{Kt} / \mathrm{V}$ & $1.54(1.30-1.71)$ & $1.52(1.35-1.78)$ & 0.3791 \\
\hline $\mathrm{nPCR}$ & $0.79(0.70-0.91)$ & $0.78(0.69-0.92)$ & 0.6712 \\
\hline TC & $151(130-184)$ & $154(136-182)$ & 0.9779 \\
\hline HDL-C & $47(39-59)$ & $53(43-59)$ & 0.0070 \\
\hline TG & $83(56-104)$ & $70(45-90)$ & 0.0515 \\
\hline $\mathrm{CPP}$ & $60,330(41,022-120,055)$ & $39,949(14,187-93,153)$ & 0.1849 \\
\hline FGF23 & $1877(486-4423)$ & $2656(444-9361)$ & 0.0142 \\
\hline \multicolumn{4}{|c|}{ JTT-751 } \\
\hline & Baseline & 24 months & $\mathrm{P}$-value \\
\hline $\mathrm{P}$ & $4.7(4.4-5.5)$ & $4.5(4.1-5.0)$ & 0.0015 \\
\hline $\mathrm{Ca}$ & $8.5(8.1-9.0)$ & $8.9(8.2-9.2)$ & 0.8628 \\
\hline iPTH & $112(67-213)$ & $183(71-266)$ & 0.0452 \\
\hline Ferritin & $116(50-234)$ & $127(71-219)$ & 0.9116 \\
\hline Hemoglobin & $11.0(10.3-11.8)$ & $11.2(9.5-12.1)$ & 0.7482 \\
\hline Albumin & $3.6(3.4-3.9)$ & $3.7(3.5-3.9)$ & 0.5367 \\
\hline CRP & $0.16(0.06-0.41)$ & $0.24(0.08-1.23)$ & 0.0699 \\
\hline $\mathrm{Kt} / \mathrm{V}$ & $1.44(1.26-1.70)$ & $1.50(1.31-1.81)$ & 0.1657 \\
\hline $\mathrm{nPCR}$ & $0.84(0.76-0.96)$ & $0.83(0.74-1.04)$ & 0.3706 \\
\hline TC & $168(143-181)$ & $160(140-186)$ & 0.5734 \\
\hline HDL & $44(36-53)$ & $49(41-61)$ & 0.0324 \\
\hline TG & $101(68-145)$ & $85(49-112)$ & 0.0250 \\
\hline $\mathrm{CPP}$ & $155,857(78,082-290,852)$ & $60,968(35,532-116,478)$ & 0.0143 \\
\hline FGF23 & $2580(830-6736)$ & $3982(1861-16,497)$ & 0.0560 \\
\hline
\end{tabular}

Data are expressed as median (interquartile rages) or numbers (\%). Ca, corrected calcium; CPP, calciprotein particle; CRP, C-reactive protein; FGF23, fibroblast growth factor 23; HDL, high-density lipoprotein cholesterol; iPTH, intact parathyroid hormone; $\mathrm{nPCR}$, normalized protein catabolic rate; $\mathrm{P}$, phosphorus; TC, total cholesterol; TG, triglycerides.

AoACS did not change significantly (from $6[5$ - 6] to 5 [5 - 9]) and $\Delta \%$ AoACS (IQR) was $0(0 \%-3 \%)$. 
Table 3. Changes in AoACS during the study period.

\begin{tabular}{|c|c|c|c|c|}
\hline \multirow[b]{2}{*}{ All participants } & \multicolumn{2}{|c|}{ JTT-751 $(\mathrm{n}=35)$} & \multicolumn{2}{|c|}{$\mathrm{CaCO}_{3}(\mathrm{n}=35)$} \\
\hline & Baseline & 24 months & Baseline & 24 months \\
\hline AoACS & $4(0-7)$ & $5(3-9)^{*}$ & $2(0-5)$ & $3(2-5)^{*}$ \\
\hline \multirow[t]{2}{*}{$\Delta \%$ AoACS } & \multicolumn{2}{|c|}{$1(0-3)$} & \multicolumn{2}{|c|}{$0(0-3)$} \\
\hline & \multicolumn{2}{|c|}{ JTT-751 $(\mathrm{n}=20)$} & \multicolumn{2}{|c|}{$\mathrm{CaCO}_{3}(\mathrm{n}=22)$} \\
\hline Baseline AoACS $\leq 4$ & Baseline & 24 months & Baseline & 24 months \\
\hline AoACS & $0(0-3)$ & $3(2-4)^{*}$ & $2(0-2)$ & $3(0-4)^{*}$ \\
\hline \multirow[t]{2}{*}{$\Delta \%$ AoACS } & \multicolumn{2}{|c|}{$2(0-3)$} & \multicolumn{2}{|c|}{$1(0-3)$} \\
\hline & \multicolumn{2}{|c|}{ JTT-751 $(\mathrm{n}=15)$} & \multicolumn{2}{|c|}{ Calcium Carbonate $(\mathrm{n}=13)$} \\
\hline Baseline AoACS $>4$ & Baseline & 24 months & Baseline & 24 months \\
\hline AoACS & $7(6-10)$ & $8(6-11)$ & $6(5-6)$ & $5(5-9)$ \\
\hline$\Delta \%$ AoACS & \multicolumn{2}{|c|}{$1(0-2)$} & \multicolumn{2}{|c|}{$0(0-3)$} \\
\hline
\end{tabular}

${ }^{\star} \mathrm{P}<0.05$ vs. baseline. Data are expressed as median (interquartile ranges). AoACS, aortic arch calcification score.

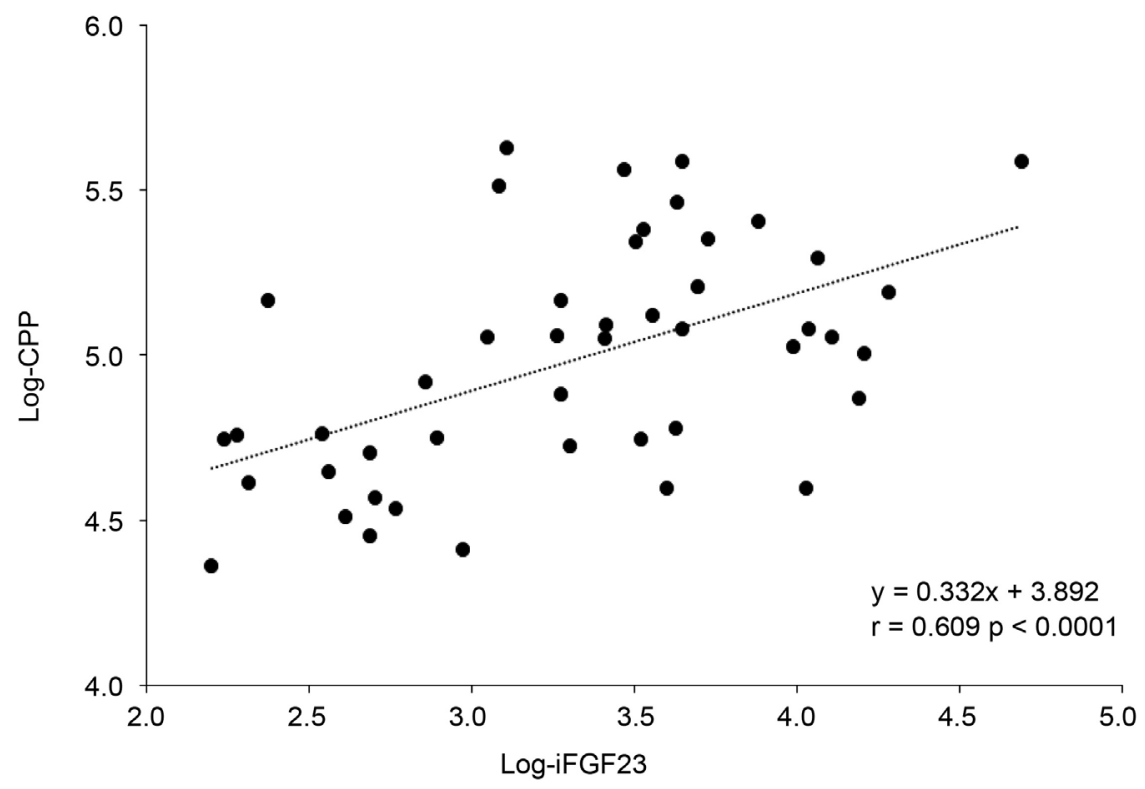

Figure 1. Correlation between serum levels of calciprotein particle (CPP) and fibroblast growth factor (FGF) 23. Values are not normally distributed and log-transformed values are shown.

In contrast, among patients with no progression of AoACS at the start of the study (initial AoACS value $\leq 4)$, median (IQR) AoACS increased from $0(0-3)$ to $3(2-4)(\mathrm{p}<0.05)$ and the $\Delta \%$ AoACS was $2(0 \%-4 \%)$ in JTT-751 group ( $\mathrm{p}=$ 0.0117). In the $\mathrm{CaCO}_{3}$ group, median AoACS increased significantly from 2 (0 2) to $3(0-4 ; \mathrm{p}=0.0073)$ and $\triangle \%$ AoACS (IQR) was $1(0 \%-3 \%)$. Figure 2 shows changes in AoACS in the JTT-751 and $\mathrm{CaCO}_{3}$ groups. 

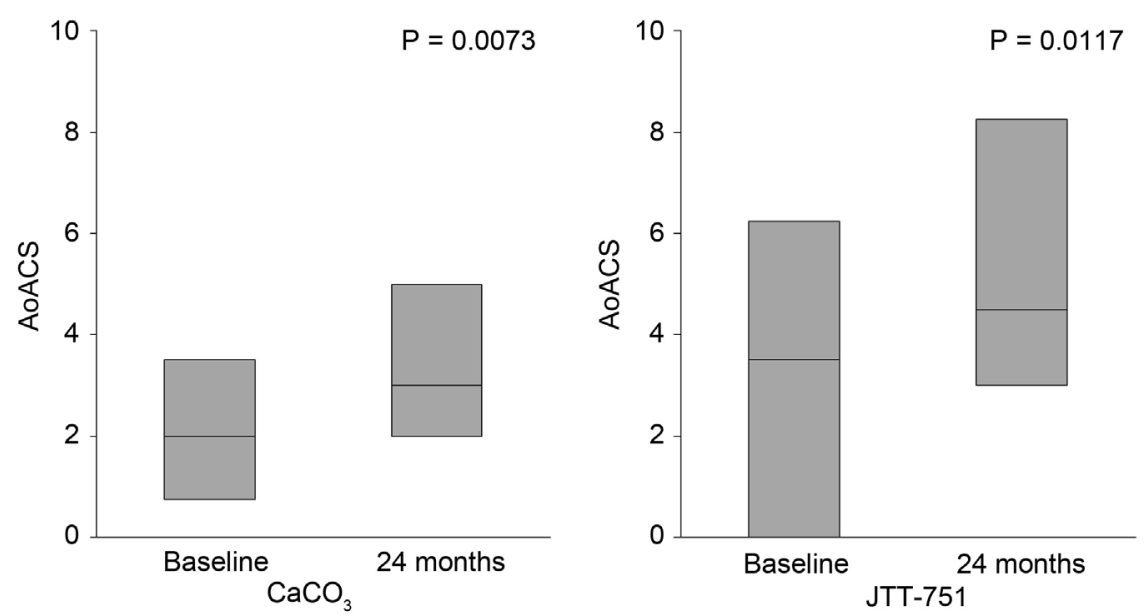

Figure 2. Changes in aortic arch calcification score (AoACS) in patients treated with $\mathrm{CaCO}_{3}$ or JTT-751. P-values are for 24-month results compared with baseline values.

\section{Discussion}

This evaluation of AoAC progression with JTT-751 compared with $\mathrm{CaCO}_{3}$ in patients on MHD is the first to our knowledge. We also showed that JTT-751 inhibits CPP production. However, JTT-751 did not suppress the progression of AoAC at 24 months after starting treatment in the entire study population. In contrast, in the group with advanced AoAC (AoACS > 4) at baseline, no significant difference was observed in the rate of AoAC progression in the JTT-751 or $\mathrm{CaCO}_{3}$ group after 24 months.

CPPs are a novel biomarker of CKD-MBD and comprise $\mathrm{P}, \mathrm{Ca}$, and proteins such as fetuin $\mathrm{A} ; \mathrm{P}$ is the main factor that contributes to CPP level and biological activity. Blood CPP levels increase with increased $\mathrm{P}$ and Ca levels, and this contributes to arteriosclerosis [7] and inflammation [8] in patients with CKD. CPP levels increase, just before the increase in FGF23 level that occurs in the G1 and G2 stages of CKD [7]. Based on the above findings, it was hypothesized that CPPs could possibly induce FGF23 and recently we confirmed this hypothesis in vitro and in vivo [17]. Moreover, CPP levels have been reported to show significant correlation with P and FGF23 levels, but not Ca levels, and thus can be increased even in of hyperphosphatemic and hypocalcemic states associated with CKD progression [9]. Nevertheless, further study is required to elucidate the mechanism underlying the formation of CPP in vivo. CPP and FGF23 could be implicated in chronological changes in CKD-MBD to facilitate homeostasis of bone and mineral metabolism.

JTT-751 is a novel P binder that has ferric citrate as the active ingredient [18] [19]. The surface area of JTT-751 is larger and it has a faster dissolution rate than ferric citrate. It is thus expected to demonstrate efficacy as a P binder and has been reported to prevent the progression of ectopic calcification, secondary hyperparathyroidism and bone disorders in rats with chronic renal failure [20]. Currently, JTT-751 is undergoing development for use in the treatment of hyperphosphatemia in dialysis patients and its efficacy and safety have been 
demonstrated in a Phase 2 dose-response study [11]. According to results of a Phase 3 clinical trial by Yokoyama et al., the safety and relative efficacy of JTT-751 was found to be comparable to that of sevelamer in patients with hyperphosphatemia on HD [21]. The use of JT-751 was associated with a significant but relative increase in serum ferritin and transferrin saturation. As a P-reducing agent, JTT-751 is well tolerated and effective and is known to have off-target effects highly likely related to reduced overall cardiovascular risk in MHD patients. In addition, JTT-751 could be an appropriate first-line treatment choice for P-reducing when indicated (serum P levels $>4.5 \mathrm{mg} / \mathrm{dL}$ ), as mentioned in the Kidney Disease: Improving Global Outcomes guidelines [5].

Transformation of primary CPP to secondary CPP metabolites depends on individual serum composition, and more precisely on the levels of calcification-inhibiting factors, including fetuin-A and albumin (proteins), and $\mathrm{Ca}, \mathrm{P}$, magnesium, and pyrophosphate (small molecules) [8] [21]. Secondary CPP metabolites induced cell necrosis, mineralization, and tumor necrosis factor (TNF)- $\alpha$ mRNA expression and protein levels in vitro using primary human aortic vascular smooth muscle cells. These findings in addition to the aggravation and amelioration of these effects by treatment with TNF- $\alpha$ and its inhibitor, respectively, suggest that secondary CPP could potentially induce local production of cytokines such as TNF- $\alpha$ with resulting vascular injury [22]. Administration of a CPP inhibitor attenuated vascular injury in rats on an adenine-containing diet and reduced blood CPP elevation and vascular calcification [23]. In contrast, secondary CPP metabolites have been identified in sites vascular injury where they are phagocytosed by CD68-positive macrophages via scavenger receptor-A I/II in ApoE and fetuin-A deficient mice [24]. CPP could thus contribute to vascular calcification in these pathological animal disease models.

AoAC is a form of vascular calcification typically seen in patients on dialysis. The progression of vascular calcification has been associated with factors including aging, diabetes mellitus, inflammation, oxidative stress, uremic toxins, the renin-angiotensin system activity, high blood pressure, hyperlipidemia, and mineral disorders [25]. Hyperphosphatemia is considered the main factor in the pathophysiology of AoAC [26]. Notably, serum P levels are prone to increase in HD patients because they cannot excrete $\mathrm{P}$ into the urine, so it is necessary to remove $\mathrm{P}$ by dialysis and control $\mathrm{P}$ burden in the intestine in these patients. However, vascular calcification is frequently observed in CKD patients with serum $P$ levels within normal reference range [27], implying that CPPs may be associated with vascular calcification. In the present study, JTT-751 reduced serum levels of $\mathrm{P}$ and $\mathrm{CPP}$, resulting in less AoAC progression despite comparable control of serum $\mathrm{P}$ and Ca levels, but only in cases with advanced AoAC at baseline. These findings suggest that JTT-751 may be effective for inhibition of vascular calcification in MHD patients [7] [8].

A large phase III trial of US patients on dialysis described the non-inferiority of the P-reducing effects of ferric citrate compared with that of sevelamer and Ca 
acetate, with a significant increase in serum transferrin saturation, ferritin, and hemoglobin levels [28] [29]. This allowed for lower requirements for intravenous iron supplementation and use of erythropoiesis-stimulating agents. These findings are consistent with reports from Japan and Taiwan [30] [31]. It has been shown that iron availability and absorption are considerably higher compared with the outcomes obtained with the use of sucroferric oxyhydroxide, and in this phase III trial hemoglobin increased by a mean of $0.5 \mathrm{~g} / \mathrm{dl}$ with comparative increases in transferrin saturation and ferritin levels.

Similar to sucroferric oxyhyroxide, ferric citrate has been linked with mild-to-moderate diarrhea and darkish discoloration of stool. The increased intestinal iron absorption is likely a result of citrate formation and the higher dose of elemental iron administered. There is current information available on exposure to ferric citrate for up to 1 year only; not much data is available regarding possibly undesirable persistent iron accumulation following long-term exposure and thus proper evaluation in the future is necessary. This agent does, however, offer the potential of effective counteract iron deficiency anemia by oral administration in patients on dialysis and may represent a $\mathrm{P}$ binder with an added value [32].

This study has some limitations. First, because of the small sample size and the relatively short observation period of 2 years, our findings should be confirmed in a larger patient group. Second, the possibility of bias due to misclassification is a key limitation that may have influenced changes in biochemical markers during the duration of treatment. Third, because treatment was not blinded the possibility of contamination and co-intervention cannot be ruled out. A new assay for measuring CPP level using fresh plasma has been developed, but the CPP levels in this study were derived from frozen serum. Fourth, in this study, we could not ascertain the actual role of JTT-751 in reducing AoAC and could not confirm an association with reduced oral $\mathrm{Ca}$ ingestion or other confounding factors. Thus, further research is warranted to clarify the role of decreased Ca intake, reduced frequency of hypercalcemic episodes, enhanced iPTH control, or other metabolic effects in the utility of JTT-751.

In conclusion, JTT-751 inhibited the production of CPP but did not suppress AoAC progression at 24 months after the start of treatment. Participants prescribed JTT-751 demonstrated less progression of AoAC even with comparable control of serum $\mathrm{P}$ or Ca levels, but only those with severe AoAC at baseline.

\section{Acknowledgements}

This study was supported in part by a grant from the Japan Research Promotion Society for Cardiovascular Disease. The funding source played no role in interpretation of the data or the preparation of the manuscript.

\section{Conflicts of Interest}

The authors declare no conflicts of interest regarding the publication of this paper. 


\section{Disclosure}

The authors have no conflict of interests to declare.

\section{References}

[1] Hruska, K.A. and Mathew, S. (2011) The Roles of the Skeleton and Phosphorus in the CKD Mineral Bone Disorder. Advances in Chronic Kidney Disease, 18, 98-104. https://doi.org/10.1053/j.ackd.2011.01.001

[2] Taniguchi, M., Fukagawa, M., Fujii, N., Hamano, T., Shoji, T., Yokoyama, K., et al. (2013) Serum Phosphate and Calcium Should Be Primarily and Consistently Controlled in Prevalent Hemodialysis Patients. Therapeutic Apheresis and Dialysis, 17, 221-228. https://doi.org/10.1111/1744-9987.12030

[3] Chiu, Y.W., Teitelbaum, I., Misra, M., de Leon, E.M., Adzize, T. and Mehrotra, R. (2009) Pill Burden, Adherence, Hyperphosphatemia, and Quality of Life in Maintenance Dialysis Patients. Clinical Journal of American Society of Nephrology, 4, 1089-1096. https://doi.org/10.2215/CJN.00290109

[4] Isakova, T., Gutierrez, O.M., Chang, Y., Shah, A., Tamez, H., Smith, K., et al. (2009) Phosphorus Binders and Survival on Hemodialysis. Journal of American Society of Nephrology, 20, 388-396. https://doi.org/10.1681/ASN.2008060609

[5] Kidney Disease: Improving Global Outcomes (KDIGO) CKD-MBD Work Group (2009) KDIGO Clinical Practice Guideline for the Diagnosis, Evaluation, Prevention, and Treatment of Chronic Kidney Disease-Mineral and Bone Disorder (CKD-MBD). Kidney International Supplement, 113, S1-S130.

[6] Price, P.A., Thomas, G.R., Pardini, A.W., Fiqueira, W.F., Capulo, J.M. and Williamson, M.K. (2002) Discovery of a High Molecular Weight Complex of Calcium, Phosphate, Fetuin, and Matrix Gamma-Carboxyglutamic Acid Protein in the Serum of Etidronate-Treated Rats. Journal of Biological Chemistry, 277, 3926-3934. https://doi.org/10.1074/jbc.M106366200

[7] Hamano, T., Matsui, I., Mikami, S., Tomida, K., Fujii, N., Imai, E., et al. (2010) Fetuin-Mineral Complex Reflects Extraosseous Calcification Stress in CKD. Journal of American Society of Nephrology, 21, 1998-2007.

https://doi.org/10.1681/ASN.2009090944

[8] Smith, E.R., Ford, M.L., Tomlinson, L.A., Rajkumar, C., McMahon, L.P. and Holt, S.G. (2012) Phosphorylated Fetuin A-Containing Calciprotein Particles Are Associated with Aortic Stiffness and a Procalcific Milieu in Patients with Pre-Dialysis CKD. Nephrology Dialysis Transplantation, 27, 1957-1966.

https://doi.org/10.1093/ndt/gfr609

[9] Miura, Y., Iwazu, Y., Shiizaki, K., Akimoto, T., Kotani, K., Kurabayashi, M., et al. (2018) Identification of Plasma Calciprotein Particles with Distinct Physical Properties in Patients with Chronic Kidney Disease. Scientific Reports, 8, Article No. 1256. https://doi.org/10.1038/s41598-018-19677-4

[10] Yang, W.C., Yang, C.S., Hou, C.C., Wu, T.H., Young, E.W. and Hsu, C.H. (2002) An Open-Label, Crossover Study of a New Phosphate-Binding Agent in Haemodialysis Patients: Ferric Citrate. Nephrology Dialysis Transplantation, 17, 265-270. https://doi.org/10.1093/ndt/17.2.265

[11] Yokoyama, K., Hirakata, H., Akiba, T., Sawada, K. and Kumagai, Y. (2012) Effect of Oral JTT-751 (Ferric Citrate) on Hyperphosphatemia in Hemodialysis Patients: Results of a Randomized, Double-Blind, Placebo-Control Trial. American Journal of Nephrology, 36, 478-487. https://doi.org/10.1159/000344008

[12] Umanath, K., Sika, M., Niecestro, R., Connelly, C., Schulman, G., Koury, M.J., et al. 
(2013) Rationale and Study Design of a Three-Period, 58-Week Trial of Ferric Citrate as a Phosphate Binder in Patients with ESRD on Dialysis. Hemodialysis International, 17, 67-74. https://doi.org/10.1111/j.1542-4758.2012.00711.x

[13] Ogawa, T., Ishida, H., Matsuda, N., Fujiu, A., Matsuda, A., Ito, K., et al. (2009) Simple Evaluation of Aortic Arch Calcification by Chest Radiography in Hemodialysis Patients. Hemodialysis International, 13, 301-306. https://doi.org/10.1111/j.1542-4758.2009.00366.x

[14] Akiyama, K., Mochizuki, T., Kataoka, H., Tsuchiya, K. and Nitta, K. (2017) Fibroblast Growth Factor 23 and Soluble Klotho in Patients with Autosomal Dominant Polycystic Kidney Disease. Nephrology (Carlton), 22, 848-853. https://doi.org/10.1111/nep.12862

[15] Daugirdas, J.T. (1993) Second Generation Logarithmic Estimates of Single-Pool Variable Volume Kt/V: An Analysis of Error. Journal of American Society of Nephrology, 4, 1205-1213.

[16] Shinzato, T., Nakai, S., Fujita, Y., Takai, I., Morita, H., Nakane, K., et al. (1994) Determination of Kt/V and Protein Catabolic Rate Using Pre- and Postdialysis Blood Urea Nitrogen Concentrations. Nephron, 67, 280-290. https://doi.org/10.1159/000187980

[17] Akiyama, K., Kimura, T. and Shiizaki, K. (2018) Biological and Clinical Effects of Calciprotein Particles on Chronic Kidney Disease-Mineral and Bone Disorder. International Journal of Endocrinology, 2018, Article ID: 5282389. https://doi.org/10.1155/2018/5282389

[18] Sinsakul, M., Sika, M., Koury, M., Shapiro, W., Greene, T., Dwyer, J., et al. (2012) The Safety and Tolerability of Ferric Citrate as a Phosphate Binder in Dialysis Patients. Nephron Clinical Practice, 121, c25-c29. https://doi.org/10.1159/000341922

[19] Dwyer, J.P., Sika, M., Schulman, G., Chang, I.J., Anger, M., Smith, M., et al. (2013) Dose-Response and Efficacy of Ferric Citrate to Treat Hyperphosphatemia in Hemodialysis Patients: A Short-Term Randomized Trial. American Journal of Kidney Disease, 61, 759-766. https://doi.org/10.1053/j.ajkd.2012.11.041

[20] Iida, A., Kemmochi, Y., Kakimoto, K., Tanimoto, M., Mimura, T., Shinozaki, Y., et al. (2013) Ferric Citrate, a New Phosphate Binder, Prevents the Complications of Secondary Hyperparathyroidism and Vascular Calcification. American Journal of Nephrology, 37, 346-358. https://doi.org/10.1159/000348805

[21] Pasch, A., Fareses, S., Graber, S., Wald, J., Richtering, W., Floege, J., et al. (2012) Nanoparticle-Based Test Measures Overall Propensity for Calcification in Serum. Journal of American Society of Nephrology, 23, 1744-1752. https://doi.org/10.1681/ASN.2012030240

[22] Aghagolzadeh, P., Bachtler, M., Bijamia, R., Jackson, C., Smith, E.R., Odermatt, A., et al. (2016) Calcification of Vascular Smooth Muscle Cells Is Induced by Secondary Calciprotein Particles and Enhanced by Tumor Necrosis Factor- $\alpha$. Atherosclerosis, 251, 404-414. https://doi.org/10.1016/j.atherosclerosis.2016.05.044

[23] Matsui, I., Hamano, T., Mikami, S., Fujii, N., Takabatake, Y., Nagasawa, Y., et al. (2009) Fully Phosphorylated Fetuin-A Forms a Mineral Complex in the Serum of Rats with Adenine-Induced Renal Failure. Kidney International, 75, 915-928. https://doi.org/10.1038/ki.2008.700

[24] Herrmann, M., Schafer, C., Heiss, A., Graber, S., Kinkeldey, A., Buscher, A., et al. (2012) Clearance of Fetuin-A-Containing Calciprotein Particles Is Mediated by Scavenger Receptor-A. Circulation Research, 111, 575-584.

https://doi.org/10.1161/CIRCRESAHA.111.261479 
[25] Nitta, K., Hanafusa, N., Okazaki, M., Komatsu, M., Kawaguchi, H. and Tsuchiya, K. (2018) Association between Risk Factors Including Bone-Derived Biomarkers and Aortic Arch Calcification in Maintenance Hemodialysis Patients. Kidney Blood Pressure Research, 43, 1554-1562. https://doi.org/10.1159/000494441

[26] Ogawa, T. and Nitta, K. (2018) Pathogenesis and Management of Vascular Calcification in Patients with End-Stage Renal Disease. Contribution to Nephrology, 196, 71-77. https://doi.org/10.1159/000485702

[27] Qunibi, W.Y. (2007) Cardiovascular Calcification in Nondialyzed Patients with Chronic Kidney Disease. Seminar in Dialysis, 20, 134-138. https://doi.org/10.1111/j.1525-139X.2007.00260.x

[28] Lewis, J.B., Sika, M., Koury, M.J., Chuang, P., Schulman, G., Smith, M.T., et al. (2015) Ferric Citrate Controls Phosphorus and Delivers Iron in Patients on Dialysis. Journal of the American Society of Nephrology, 26, 493-503. https://doi.org/10.1681/ASN.2014020212

[29] Umanath, K., Jalal, D.I., Greco, B.A., Umeukeje, E.M., Reisin, E., Manley, J., et al. (2015) Ferric Citrate Reduces Intravenous Iron and Erythropoiesis-Stimulating Agent Use in ESRD. Journal of American Society of Nephrology, 26, 2578-2587. https://doi.org/10.1681/ASN.2014080842

[30] Yokoyama, K., Akiba, T., Fukagawa, M., Nakayama, M., Sawada, K., Kumagai, Y., et al. (2014) A Randomized Trial of JTT-751 versus Sevelamer Hydrochloride in Patients on Hemodialysis. Nephrology Dialysis Transplantation, 29, 478-487. https://doi.org/10.1093/ndt/gft483

[31] Lee, C.T., Wu, I.W., Chiang, S.S., Peng, Y.S., Shu, K.H., Wu, M.J., et al. (2015) Effect of Oral Ferric Citrate on Serum Phosphorus in Hemodialysis Patients: Multicenter, Randomized, Double-Blind, Placebo-Controlled Study. Journal of Nephrology, 28, 105-113. https://doi.org/10.1007/s40620-014-0108-6

[32] Ketteler, M., Liangos, O. and Biggar, P.H. (2016) Treating Hyperphosphatemia-Current and Advancing Drugs. Expert Opinion on Pharmacotherapy, 17, 1873-1879. https://doi.org/10.1080/14656566.2016.1220538 